\title{
Antineuropathic and Antinociceptive Drugs Combination in Patients with Chronic Low Back Pain: A Systematic Review
}

\author{
Carlo Luca Romanò, ${ }^{1}$ Delia Romanò, ${ }^{1}$ and Marco Lacerenza ${ }^{2}$ \\ ${ }^{1}$ Centro di Chirurgia Ricostruttiva, Istituto Ortopedico I.R.C.C.S. Galeazzi, Via R. Galeazzi 4, 20161 Milano, Italy \\ ${ }^{2}$ Centro di Medicina del Dolore, Casa di cura S. Pio X, Fondazione Opera San Camillo, Via F. Nava 31, 20159 Milano, Italy \\ Correspondence should be addressed to Carlo Luca Romanò, carlo.romano@grupposandonato.it
}

Received 8 January 2012; Revised 4 February 2012; Accepted 8 February 2012

Academic Editor: Young-Chang P. Arai

Copyright ( 92012 Carlo Luca Romanò et al. This is an open access article distributed under the Creative Commons Attribution License, which permits unrestricted use, distribution, and reproduction in any medium, provided the original work is properly cited.

\begin{abstract}
Purpose. Chronic low back pain (LBP) is often characterized by both nociceptive and neuropathic components. While various monotherapies have been reported of only limited efficacy, combining drugs with different mechanisms of action and targets appears a rational approach. Aim of this systematic review is to assess the efficacy and safety of different combined pharmacological treatments, compared to monotherapy or placebo, for the pharmacological treatment of chronic LBP. Methods. Published papers, written or abstracted in English from 1990 through 2011, comparing combined pharmacological treatments of chronic LBP to monotherapy or placebo were reviewed. Results. Six articles met the inclusion criteria. Pregabalin combined with celecoxib or opioids was shown to be more effective than either monotherapy. Oxycodone-paracetamol versus previous treatments and tramadol-paracetamol versus placebo were also reported as effective, while morphine-nortriptyline did not show any benefit over any single agent. Conclusions. In spite of theoretical advantages of combined pharmacological treatments of chronic LBP, clinical studies are remarkably few. Available data show that combined therapy, including antinociceptive and antineuropathic agents is more effective than monotherapy, with similar side effects.
\end{abstract}

\section{Introduction}

Successful treatment of chronic pain depends on identification of the involved mechanism and use of appropriate therapeutic approaches. Woolf et al. [1] proposed that pain symptoms and syndromes should be classified into two broad mechanism-based pain categories: tissue-injury pain (nociceptive) or nervous-system-injury pain (neuropathic).

Even if there is increasing knowledge that different mechanisms of pain require appropriate treatments and often polypharmacotherapy, and although drug combination is frequently empirically adopted in the clinical practice [2-5], prospective studies concerning the relative efficacy and safety of therapeutical drug associations to treat various painful conditions are still remarkably few [6-10] and, as recently reported, "more preclinical, clinical, and translational studies are needed to improve the efficacy of combination drug therapy that is an integral part of a comprehensive approach to the management of chronic pain" [11].
Although many patients have self-limited episodes of acute low-back pain (LBP) and do not seek medical care [12], this condition is among the five most common reasons for all physician visits in the USA $[13,14]$. Among those who do seek medical care, pain, disability, and return to work typically improve rapidly in the first month [15]; however, up to one-third of patients report persistent back pain of at least moderate intensity one year after an acute episode $[16,17]$.

Medications are the most frequently recommended intervention for low back pain $[14,18]$. In one study, $80 \%$ of primary care patients with low back pain were prescribed at least one medication at their initial office visit, and more than onethird were prescribed two or more drugs [5]. The most commonly prescribed medications for low back pain are nonsteroidal anti-inflammatory drugs (NSAIDs), skeletal muscle relaxants, and opioid analgesics $[5,19,20]$. Benzodiazepines, systemic corticosteroids, antidepressant medications, and antiepileptic drugs are also prescribed [21]. Monotherapies of chronic LBP with NSAIDs, acetaminophen and tricyclic 


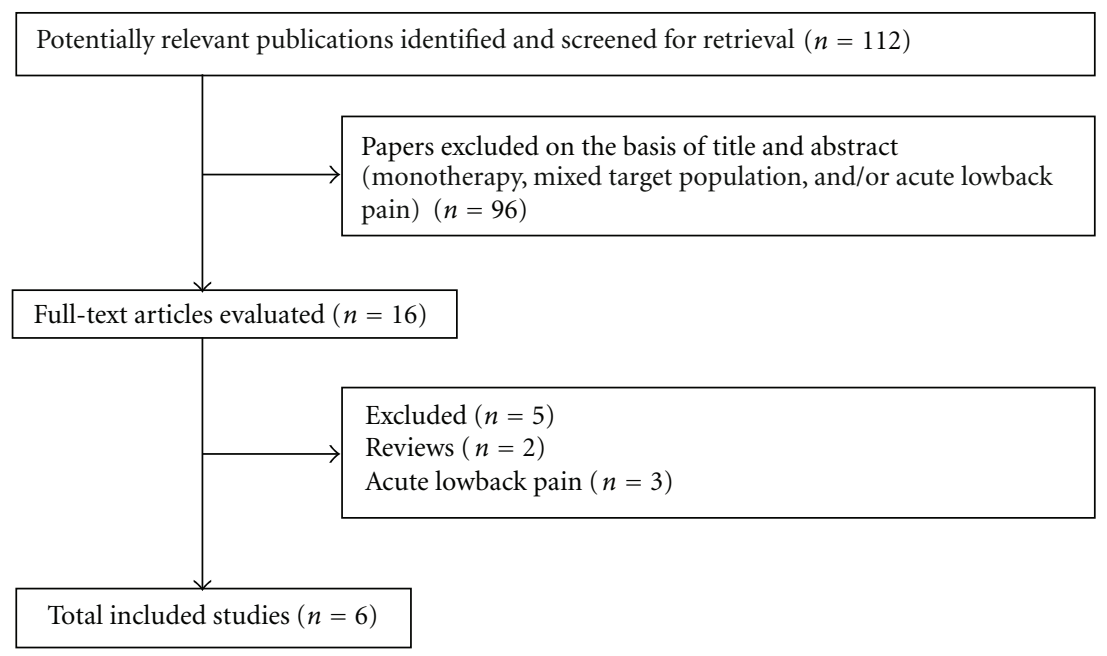

Figure 1: Flow diagram of inclusion and exclusion of articles for combined pharmacological interventions for chronic low back pain.

antidepressants, opioids, tramadol, benzodiazepines, and gabapentin (for radiculopathy) have all been found to provide only a limited pain relief, ranging from 10 to 20 points on a 100-point visual analogue pain scale [22].

Chronic LBP has been shown to be the result of neuropathic as well as nociceptive pain mechanisms and has therefore been classified as a mixed pain syndrome [23-25]. Nonspecific nociceptive pain is the result of an inflammatory response to tissue injury, while neuropathic pain describes cutaneous projected pain arising from the lumbar spine and/or nerve roots (radicular pain or radiculopathy) $[3,4]$. The multifactorial nature of chronic LBP has often been underrecognized and undertreated. Thus, recent studies have demonstrated that approximately $20-55 \%$ of patients with chronic LBP have a $>90 \%$ likelihood of a neuropathic pain component and, in an additional $28 \%$ of patients, a neuropathic pain component is suspected $[5,26,27]$. The presence of a neuropathic pain component is associated with more severe pain symptoms and higher healthcare utilization costs [28].

Based on this evidence, it has been suggested that antidepressants and/or anticonvulsants in combination with either opioids, traditional nonsteroidal anti-inflammatory drugs, or muscle relaxants could be useful in the treatment of this condition $[27,29,30]$. The aim of this systematic review is to evaluate evidence for the effectiveness of pharmacological combination therapy in chronic LBP, with specific reference to the management of nociceptive and neuropathic pain components.

\section{Materials and Methods}

Published papers written in English or including an English abstract, published from 1990 through 2011 and reporting the results of a combined pharmacological treatment of chronic lowback pain (LPB), compared with monotherapy or placebo, were reviewed. To this aim, we searched international databases, including EMBASE, PubMed/Medline, Google Scholar, SCOPUS, CINAHL, Cochrane Central Re- gister of Controlled Trials, Cochrane Database of Systematic Reviews, http://www.google.com/, and http://www .yahoo.com/. Inclusion criteria were the following:

(a) papers written or with an abstract in English;

(b) papers concerning the results of management of chronic low-back pain (symptoms duration $>6$ months);

(c) treatment using a combination of two or more drugs, versus monotherapy or placebo.

Two investigators, CR and ML, searched and reviewed independently the literature and classified the references found in terms of whether they should be included on basis of the title and the abstract of the paper. In addition to original study reports, review articles were also included and the reference lists from all reviewed articles were assessed to complete the literature search. At the end of the reviewing process, the two reviewers' lists of papers were compared and if any discrepancy occurred, reclassification was performed according to the consensus reached.

This strategy identified 112 articles, the abstracts of which were hand searched to identify a subset with the specific focus of pharmacological treatment of chronic LBP of relevance to the current review. Six studies on pharmacological management of chronic LBP (irrespective of the cause) were identified as relevant and were included in this paper (Figure 1).

\section{Results}

Table 1 summarizes the included studies examining combination pharmacotherapy of chronic LBP. Three studies evaluated paracetamol in combination with tramadol [35, 36] or oxycodone [32].

In the first study $(n=318)$, three-month treatment with tramadol $37.5 \mathrm{mg} /$ paracetamol $325 \mathrm{mg}$ yielded significantly greater improvements in pain VAS score $(P<0.015)$ and Pain Relief Rating Scale score $(P<0.001)$ than placebo. 


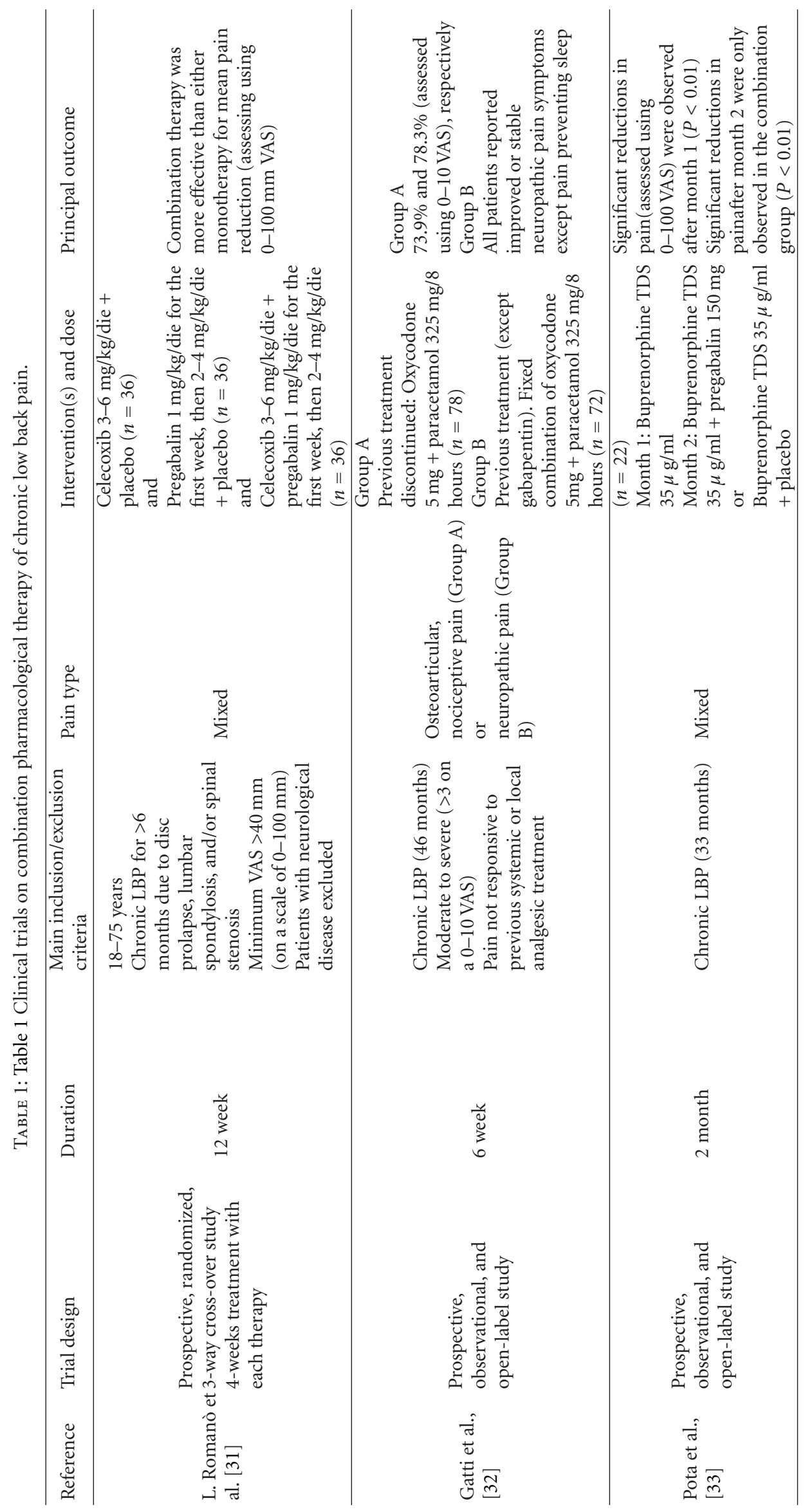




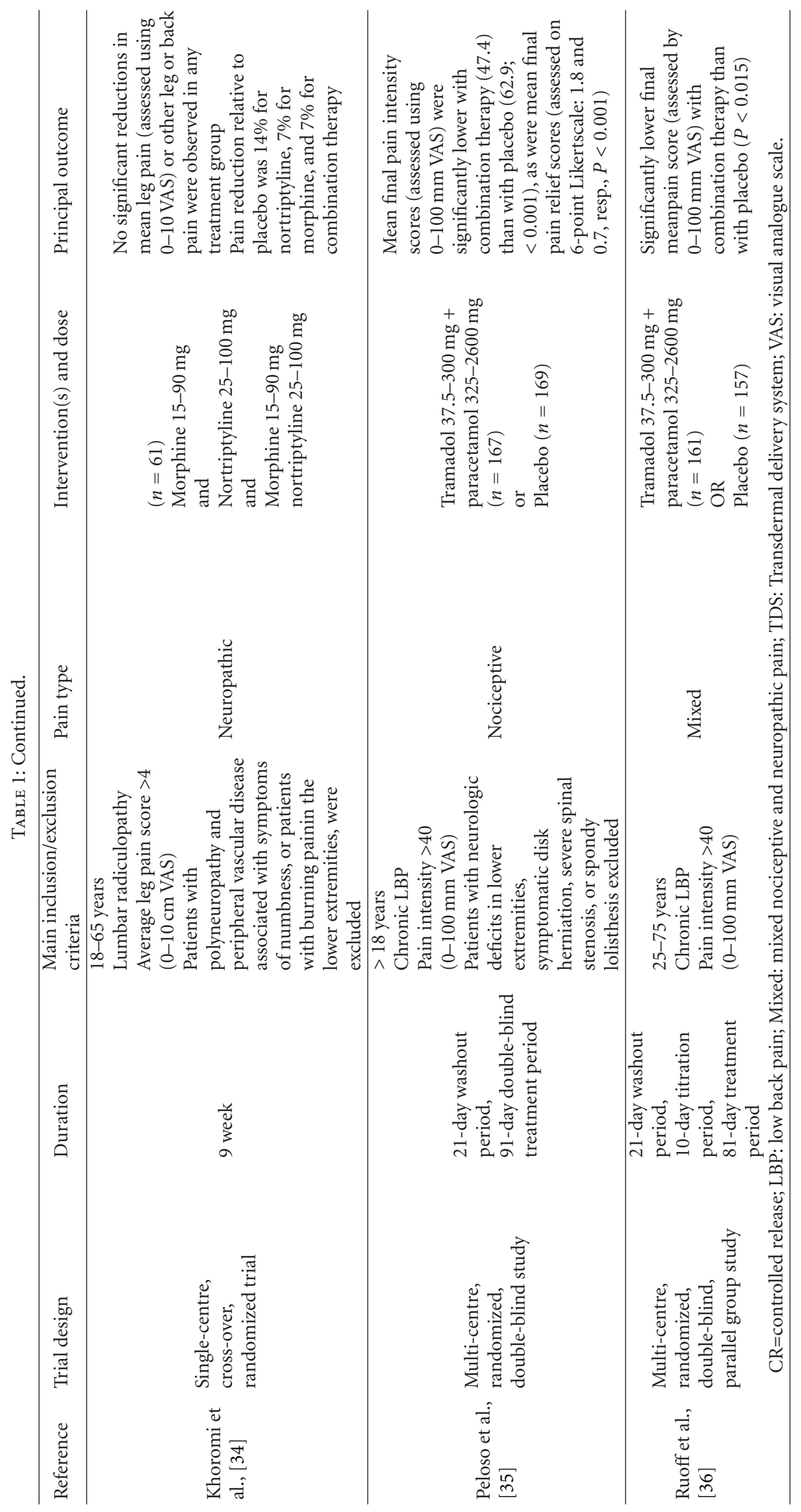


Significant improvements were also observed for Roland Disability Questionnaire (RDQ) scores, several of the sensory Short-Form McGill Pain Questionnaire (SF-MPQ) items, and the Role-Physical, Bodily Pain, Role-Emotional, Mental Health, Reported Health Transition and Mental Component items of the Short Form 36 (SF 36; all $P<0.05$ ). The rates of discontinuation due to insufficient pain relief were significantly lower with tramadol plus paracetamol (22.1\%) than for placebo $(41.0 \% ; P<0.001)$, and the proportion of patients and investigators rating treatment as "good" or "very good" was higher with combination therapy than with placebo ( $P<0.001$ for patients; $P=0.002$ for investigators). Adverse events were more common with the combination $(68.9 \%)$ than with placebo $(46.5 \%)$, as were adverse drug reactions $(23.6 \%$ versus $3.8 \%)$ and rates of discontinuation due to adverse events (18.6\% versus 5.7\%). Nausea, somnolence, and constipation were significantly more frequent with combination treatment than with placebo $(P<0.05)$ [36].

In the second study, patients with at least moderate chronic LBP received tramadol $37.5 \mathrm{mg} /$ paracetamol $325 \mathrm{mg}$ in a fixed combination tablet; VAS scores after 3 months were significantly lower with tramadol/paracetamol than with placebo $(P<0.001)$. Combination therapy was also associated with significantly improved scores on several measures, including RDQ score and physical-related items on the SF-MPQ and SF-36 $(P<0.05)$. Similar results to those reported above by Ruoff et al. [36] were observed for discontinuation due to insufficient pain relief, the proportion of patients rating treatment as "good" or "very good" and the incidence of adverse events [35].

Gatti et al., in a prospective observational study [32] examined the efficacy of a fixed-dose combination of oxycodone plus paracetamol using the Pain Management Index. Patients were stratified according to the presence of prevalent osteoarticular pain $(n=78)$ or prevalent neuropathic pain $(n=72)$. Combination therapy was associated with an improvement in pain in the majority of compliant patients, although its benefit in patients with neuropathic pain was less marked.

Two papers reported on the efficacy of pregabalin with, respectively, celecoxib [31] or transdermal (TDS) buprenorphine [33]. In our previously published prospective, singleblind, randomized study [31], the safety and efficacy of the association of celecoxib and pregabalin with either monotherapy for treatment of chronic low back pain of various origin, were compared; data were also analyzed on the basis of pain quality assessed with the Leeds Assessment of Neuropathic Symptoms and Signs (LANSS) pain scale [37, 38]. Our study showed that the association pregabalin/celecoxib resulted in a statistically significant reduction of self-reported pain when considering either all the recruited patients or the subpopulations divided according to LANSS score. On the contrary, celecoxib/placebo and pregabalin/placebo only produced a statistically significant reduction of reported pain in, respectively, patients with LANSS score $<12(P=0.01)$ (nociceptive pain) and in patients with LANSS score $>12$ $(P=0.03)$ (neuropathic pain), but not when including all the patients. The drug combination also proved to be more effective than pregabalin alone or than celecoxib alone, except for patients with LANSS score $<12$, in which treatment combination or monotherapy provided similar results. When all patients were considered, celecoxib alone provided $12.4 \%$ pain reduction, pregabalin alone $10.4 \%$, and their combination $38.2 \%$. The largest pain reduction (51.8\%) was observed with the association pregabalin/celecoxib in patients with LANSS score $>12$. Pregabalin drug consumption, when used in association with celecoxib, was significantly lower $(P<0.05)$ compared to monotherapy. The occurrence of side effects was similar during either monotherapy or combination treatment [31].

Similarly, Pota and coworkers [33] found that the combination of pregabalin and buprenorphine, TDS yielded significantly greater reductions in VAS scores than buprenorphine monotherapy $(P<0.01)$. In the first month of therapy buprenorphine TDS alone provided a meaningful pain reduction (VAS $82.75 \pm 15$ versus $38.25 \pm 5, P<0.01$ ); at the end of the first month, patients were then divided in two groups: Group A receiving one-month therapy with buprenorphine $35 \mu \mathrm{g} / \mathrm{mL}$ plus pregabalin $150 \mathrm{mg}$ and Group $B$ buprenorphine plus placebo. At the end of the treatment, only Group A presented a further reduction of the VAS $(P<0.01)$. The authors concluded that "buprenorphine TDS determines a notable relief from pain. Moreover the association of low doses of pregabalin allowed a further relief."

The unique study evaluating the combination of morphine with nortriptyline [34], failed to provide sufficient data as to regard the efficacy of this free opioid-antidepressant combination for the treatment of chronic LBP. In this study, performed on 61 patients with sciatica, the combination of morphine and nortriptyline did not reduce average leg pain scores or any other leg or back pain scores, while $89 \%$ of patients receiving combination treatment reported an adverse event, most commonly constipation.

\section{Discussion}

This systematic review shows that, in spite chronic LBP is thought to be commonly the result of both nociceptive and neuropathic mechanisms [23] and hence a rationale approach would be targeting the different mechanisms of pain by combining specific drug agents, remarkably few clinical trials are currently available to validate this hypothesis.

This may due to different reasons including

(i) the difficulty in designing/performing clinical trials involving more treatments at the same time;

(ii) potential drugs' interactions and possible adverse effects. Any specific combination of agents need to be first evaluated on the basis of the respective pharmacokinetic profile and possible interactions and then clinically tested. In free dose combinations, the onset of adverse events can, to some extent, be overcome by initiating treatment at low doses and slowly escalating the dose until maximum analgesia or intolerable side effects arise; drug combination may also provide reduced consumption of any single drug and adverse events comparable to monotherapy [31]; 
(iii) unpredictable dosing regimen. At variance with that reported above concerning the possible advantage of free dose combinations, fixed dose are easier to study and to market, being also likely associated with greater patient's compliance than free combinations; however, identifying the best dose ratio for all the patients, balancing the efficacy and tolerability of any single drug within a fixed combination may be a challenging exercise;

(iv) scarce economical interest of drug companies.

All of these potential drawbacks may, to a different extent, concur to explain the limited research on drug combinations, in spite of theoretical positive considerations and notwithstanding the empirical widespread use of drug associations in the clinical practice [5].

Among the six studies that were included in the present review, two examined a fixed-dose regimen of paracetamol and tramadol combination against placebo $[35,36]$. These studies appear much similar, in their design and outcomes, to a "traditional" monotherapy versus placebo study [39] and do not really seem to add any insight as to concern the control of different types of pain.

On the contrary, the association of pregabalin plus celecoxib [31] or of pregabalin and an opioid agent [33] seem more focused on targeting different pain components of chronic LBP.

Gabapentinoids have already been successfully used in combination with other analgesic drugs to improve neuropathic pain control. Gilron et al. [7] first reported on the efficacy and safety of a combination of gabapentin and morphine compared with that of each as a single agent in patients with painful diabetic neuropathy or postherpetic neuralgia. In 41 patients, gabapentin-morphine combination showed significantly better pain control $(P<0.05)$ versus placebo, gabapentin, and morphine. More recently, Gatti et al. reported the Multicenter Italian Study, which compared the efficacy, safety, and quality of life of combination therapy with controlled release (CR) oxycodone plus pregabalin versus monotherapy in patients with neuropathic pain of various origins [40]. This study showed in 409 patients that the combination of CR oxycodone plus pregabalin was more effective than monotherapy for alleviating neuropathic pain $(P=0.003)$ and to improve quality of life $(P=0.0009)$, while combination therapy also allowed dose reduction of both agents (22\% for CR oxycodone and $51 \%$ for pregabalin).

Interestingly, in our reported study, celecoxib or pregabalin when used alone were shown to be not effective in patients with, respectively, neuropathic or nociceptive low back pain type, as evaluated with the LANSS pain scale [31]. This is not surprising, given the specific ability of pregabalin to control neuropathic pain $[2,41,42]$, while celecoxib is a selective COX-2 inhibitor that has been proved to be effective in the treatment of different pain models that are considered predominantly of nociceptive origin $[43,44]$. However, this finding also supports the hypothesis of a better efficacy of a combined approach to the mixed pain conditions and points out the importance of patient selection when evaluating the analgesic efficacy of any specific treatment.

A recent systematic review of pharmacological monotherapies for chronic nonspecific low back pain [45] showed no effects of different types of antidepressants, compared to placebo, on any of the primary investigated outcomes, including pain intensity, depression and functional status. The study from Khoromi et al. [34], in a mixed pain population, suffering from low back pain with lumbar radiculopathy, seems to confirm, with the limitation imposed by the small sample size, that even nortriptyline alone or in combination with morphine has limited effectiveness.

Other frequently prescribed medications, like muscle relaxants $[19,20]$, have not been investigated in randomized clinical trials for the treatment of chronic low-back pain [45] and we could not find any study regarding their use in a combined pharmacological therapy of this condition.

It is worth noting how published comprehensive reviews of clinical trials [46] and even the most recently reported guidelines concerning the treatment of chronic low back pain fail to address the use of combined pharmacological treatments $[47,48]$. While, in fact, several drugs are compared and recommended as monotherapy, associations are not mentioned. The paucity of the available data may wellexplain, in our opinion, the lack of indications in this regard and points out the need for further research and well designed clinical trials.

\section{Conclusions}

Pain treatment should be guided by the underlying mechanisms and should take into consideration pain quality as well as pain intensity. Chronic LBP often comprises both nociceptive and neuropathic components, and various monotherapies have been repeatedly reported as only partially effective. Therefore, an individualized, multimodal therapy, combining drugs with different mechanisms of action represents a rational approach. However, available studies investigating drug combinations are remarkably few. In particular, combination of pregabalin and celecoxib or buprenorphine has been demonstrated to be more effective that either monotherapy and relatively safe. The association of paracetamol with tramadol or oxycodone has also been shown to be effective for reducing chronic low-back pain, even if not evaluated against respective monotherapy. Further research in combined pharmacological treatment of chronic LBP with well-designed studies may offer valuable tools for the clinical practice and is strongly suggested.

\section{Conflict of Interests}

The authors declare that they have no conflict of interest related to the publication of this paper.

\section{References}

[1] C. J. Woolf, G. J. Bennett, M. Doherty et al., "Towards a mechanism-based classification of pain?” Pain, vol. 77, no. 3, pp. 227-229, 1998. 
[2] G. Cruccu, "Treatment of painful neuropathy," Current Opinion in Neurology, vol. 20, no. 5, pp. 531-535, 2007.

[3] G. Forde, "Adjuvant analgesics for the treatment of neuropathic pain: evaluating efficacy and safety profiles," Journal of Family Practice, pp. 3-12, 2007.

[4] C. J. Woolf, "Pain: moving from symptom control toward mechanism-specific pharmacologic management," Annals of Internal Medicine, vol. 140, no. 6, pp. 441-451, 2004.

[5] D. C. Cherkin, K. J. Wheeler, W. Barlow, and R. A. Deyo, "Medication use for low back pain in primary care," Spine, vol. 23, no. 5, pp. 607-614, 1998.

[6] I. Gilron, E. Orr, D. Tu, J. Peter O’Neill, J. E. Zamora, and A. C. Bell, "A placebo-controlled randomized clinical trial of perioperative administration of gabapentin, rofecoxib and their combination for spontaneous and movement-evoked pain after abdominal hysterectomy," Pain, vol. 113, no. 1-2, pp. 191-200, 2005.

[7] I. Gilron, J. M. Bailey, D. Tu, R. R. Holden, D. F. Weaver, and R. L. Houlden, "Morphine, gabapentin, or their combination for neuropathic pain," The New England Journal of Medicine, vol. 352, no. 13, pp. 1324-1334, 2005.

[8] J. A. Haythornthwaite, "Clinical trials studying pharmacotherapy and psychological treatments alone and together," Neurology, vol. 65, no. 12, pp. S20-S31, 2005.

[9] N. Torrance, B. H. Smith, M. C. Watson, and M. I. Bennett, "Medication and treatment use in primary care patients with chronic pain of predominantly neuropathic origin," Family Practice, vol. 24, no. 5, pp. 481-485, 2007.

[10] B. Morlion, "Pharmacotherapy of low back pain: targeting nociceptive and neuropathic pain components," Current Medical Research and Opinion, vol. 27, no. 1, pp. 11-33, 2011.

[11] J. Mao, M. S. Gold, and M. M. Backonja, "Combination drug therapy for chronic pain: a call for more clinical studies," Journal of Pain, vol. 12, no. 2, pp. 157-166, 2011.

[12] T. S. Carey, A. T. Evans, N. M. Hadler et al., "Acute severe low back pain: a population-based study of prevalence and careseeking," Spine, vol. 21, no. 3, pp. 339-344, 1996.

[13] R. A. Deyo, S. K. Mirza, and B. I. Martin, "Back pain prevalence and visit rates: estimates from U.S. national surveys, 2002," Spine, vol. 31, no. 23, pp. 2724-2727, 2006.

[14] L. G. Hart, R. A. Deyo, and D. C. Cherkin, "Physician office visits for low back pain: frequency, clinical evaluation, and treatment patterns from a U.S. National survey," Spine, vol. 20, no. 1, pp. 11-19, 1995.

[15] L. H. M. Pengel, R. D. Herbert, C. G. Maher, and K. M. Refshauge, "Acute low back pain: systematic review of its prognosis," British Medical Journal, vol. 327, no. 7410, pp. 323-325, 2003.

[16] J. W. Frymoyer and W. L. Cats-Baril, "An overview of the incidences and costs of low back pain," Orthopedic Clinics of North America, vol. 22, no. 2, pp. 263-271, 1991.

[17] M. Von Korff and K. Saunders, "The course of back pain in primary care," Spine, vol. 21, no. 24, pp. 2833-2839, 1996.

[18] M. T. Vogt, C. K. Kwoh, D. K. Cope, T. A. Osial, M. Culyba, and T. W. Starz, "Analgesic usage for low back pain: impact on health care costs and service use," Spine, vol. 30, no. 9, pp. 1075-1081, 2005.

[19] E. Bernstein, T. S. Carey, and J. M. Garrett, "The use of muscle relaxant medications in acute low back pain," Spine, vol. 29, no. 12, pp. 1346-1351, 2004.

[20] X. Luo, R. Pietrobon, L. H. Curtis, and L. A. Hey, "Prescription of nonsteroidal anti-inflammatory drugs and muscle relaxants for back pain in the United States," Spine, vol. 29, no. 23, pp. E531-E537, 2004.
[21] D. Di Iorio, E. Henley, and A. Doughty, "A survey of primary care physician practice patterns and adherence to acute low back problem guidelines," Archives of Family Medicine, vol. 9, no. 10, pp. 1015-1021, 2000.

[22] R. Chou and L. H. Huffman, "Medications for acute and chronic low back pain: a review of the evidence for an American Pain Society/American College of Physicians clinical practice guideline," Annals of Internal Medicine, vol. 147, no. 7, pp. 505-514, 2007.

[23] R. Baron and A. Binder, "Is sciatica neuropathic? The mixed pain concept," Orthopade, vol. 33, no. 5, pp. 568-575, 2004.

[24] R. Freynhagen, R. Baron, T. Tölle et al., "Screening of neuropathic pain components in patients with chronic back pain associated with nerve root compression: a prospective observational pilot study (MIPORT)," Current Medical Research and Opinion, vol. 22, no. 3, pp. 529-537, 2006.

[25] R. Freynhagen, R. Rolke, R. Baron et al., "Pseudoradicular and radicular low-back pain-a disease continuum rather than different entities? Answers from quantitative sensory testing," Pain, vol. 135, no. 1-2, pp. 65-74, 2008.

[26] A. E. Hassan, H. A. Saleh, Y. M. Baroudy et al., "Prevalence of neuropathic pain among patients suffering from chronic low back pain in Saudi Arabia," Saudi Medical Journal, vol. 25, no. 12, pp. 1986-1990, 2004.

[27] A. M. Kaki, A. Z. El-Yaski, and E. Youseif, "Identifying neuropathic pain among patients with chronic low-back pain: use of the Leeds Assessment of Neuropathic Symptoms and Signs pain scale," Regional Anesthesia and Pain Medicine, vol. 30, no. 5, pp. 422.e1-422.e9, 2005.

[28] C. O. Schmidt, B. Schweikert, C. M. Wenig et al., "Modelling the prevalence and cost of back pain with neuropathic components in the general population," European Journal of Pain, vol. 13, no. 10, pp. 1030-1035, 2009.

[29] N. B. Finnerup, M. Otto, H. J. McQuay, T. S. Jensen, and S. H. Sindrup, "Algorithm for neuropathic pain treatment: an evidence based proposal," Pain, vol. 118, no. 3, pp. 289-305, 2005.

[30] M. H. Moskowitz, "Pharmacotherapy neuropathic low back pain," Current Pain and Headache Reports, vol. 7, no. 3, pp. 178-187, 2003.

[31] C. L. Romanò, D. Romanò, C. Bonora, and G. Mineo, "Pregabalin, celecoxib, and their combination for treatment of chronic low-back pain," Journal of Orthopaedics and Traumatology, vol. 10, no. 4, pp. 185-191, 2009.

[32] A. Gatti, A. F. Sabato, A. Carucci, L. Bertini, M. Mammucari, and R. Occhioni, "Adequacy assessment of oxycodone/paracetamol (acetaminophen) in multimodal chronic pain: a prospective observational study," Clinical Drug Investigation, vol. 29, supplement 1, pp. 31-40, 2009.

[33] V. Pota, M. Maisto, M. C. Pace et al., "191 Association of buprenorphine TDS and pregabalin in the treatment of low back pain," European Journal of Pain, vol. 11, no. S1, p. S83, 2007.

[34] S. Khoromi, L. Cui, L. Nackers, and M. B. Max, "Morphine, nortriptyline and their combination vs. placebo in patients with chronic lumbar root pain," Pain, vol. 130, no. 1-2, pp. 66-75, 2007.

[35] P. M. Peloso, L. Fortin, A. Beaulieu, M. Kamin, and N. R. Rosenthal, "Analgesic efficacy and safety of tramadol/acetaminophen combination tablets (Ultracet) in treatment of chronic low back pain: a multicenter, outpatient, randomized, double blind, placebo controlled trial," Journal of Rheumatology, vol. 31, no. 12, pp. 2454-2463, 2004. 
[36] G. E. Ruoff, N. Rosenthal, D. Jordan, R. Karim, and M. Kamin, "Tramadol/acetaminophen combination tablets for the treatment of chronic lower back pain: a multicenter, randomized, double-blind, placebo-controlled outpatient study," Clinical Therapeutics, vol. 25, no. 4, pp. 1123-1141, 2003.

[37] M. Bennett, "The LANSS Pain Scale: the Leeds assessment of neuropathic symptoms and signs," Pain, vol. 92, no. 1-2, pp. 147-157, 2001.

[38] C. Pérez, R. Gálvez, J. Insausti et al., "Linguistic adaptation and Spanish validation of the LANSS (Leeds Assessment of Neuropathic Symptoms and Signs) scale for the diagnosis of neuropathic pain," Medicina Clinica, vol. 127, no. 13, pp. 485491, 2006.

[39] T. J. Schnitzer, W. L. Gray, R. Z. Paster, and M. Kamin, "Efficacy of tramadol in treatment of chronic low back pain," Journal of Rheumatology, vol. 27, no. 3, pp. 772-778, 2000.

[40] A. Gatti, A. F. Sabato, R. Occhioni, G. Colini Baldeschi, and C. Reale, "Controlled-release oxycodone and pregabalin in the treatment of neuropathic pain: results of a multicenter Italian study," European Neurology, vol. 61, no. 3, pp. 129-137, 2009.

[41] H. Lesser, U. Sharma, L. LaMoreaux, and R. M. Poole, "Pregabalin relieves symptoms of painful diabetic neuropathy: a randomized controlled trial," Neurology, vol. 63, no. 11, pp. 2104-2110, 2004.

[42] R. W. Richter, R. Portenoy, U. Sharma, L. Lamoreaux, H. Bockbrader, and L. E. Knapp, "Relief of painful diabetic peripheral neuropathy with pregabalin: a randomized, placebocontrolled trial," Journal of Pain, vol. 6, no. 4, pp. 253-260, 2005.

[43] A. Meunier, B. Lisander, and L. Good, "Effects of celecoxib on blood loss, pain, and recovery of function after total knee replacement: a randomized placebo-controlled trial," Acta Orthopaedica, vol. 78, no. 5, pp. 661-667, 2007.

[44] G. Singh, J. G. Fort, J. L. Goldstein et al., "Celecoxib versus naproxen and diclofenac in osteoarthritis patients: SUCCESSI study," American Journal of Medicine, vol. 119, no. 3, pp. 255266, 2006.

[45] T. Kuijpers, M. van Middelkoop, S. M. Rubinstein et al., "A systematic review on the effectiveness of pharmacological interventions for chronic non-specific low-back pain," European Spine Journal, vol. 20, no. 1, pp. 40-50, 2010.

[46] T. J. Schnitzer, A. Ferraro, E. Hunsche, and S. X. Kong, "A comprehensive review of clinical trials on the efficacy and safety of drugs for the treatment of low back pain," Journal of Pain and Symptom Management, vol. 28, no. 1, pp. 72-95, 2004.

[47] R. Chou, A. Qaseem, V. Snow et al., "Diagnosis and treatment of low back pain: a joint clinical practice guideline from the American College of Physicians and the American Pain Society," Annals of Internal Medicine, vol. 147, no. 7, pp. 478491, 2007.

[48] A. P. White, P. M. Arnold, D. C. Norvell, E. Ecker, and M. G. Fehlings, "Pharmacologic management of chronic low back pain: synthesis of the evidence," Spine, vol. 36, supplement 21, pp. S131-S143, 2011. 


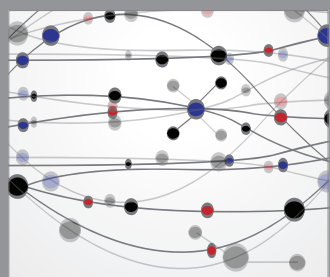

The Scientific World Journal
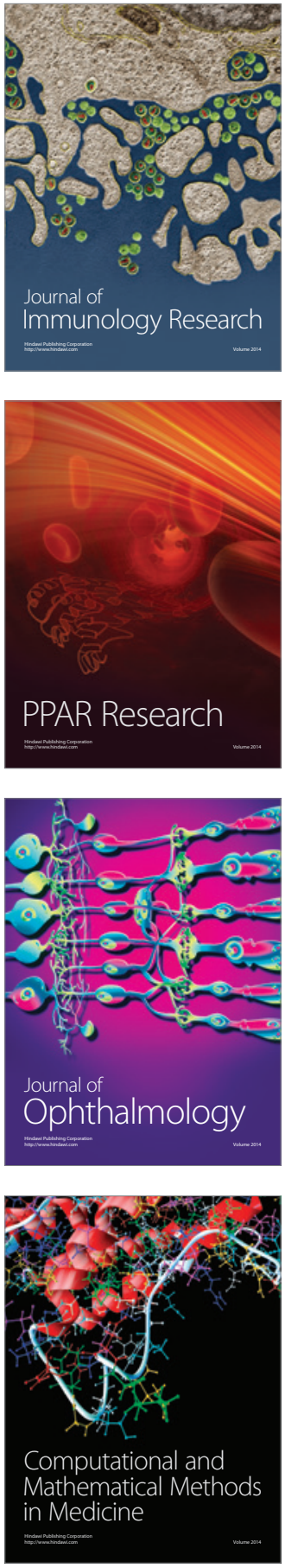

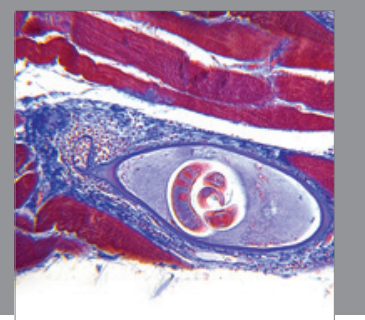

Gastroenterology

Research and Practice
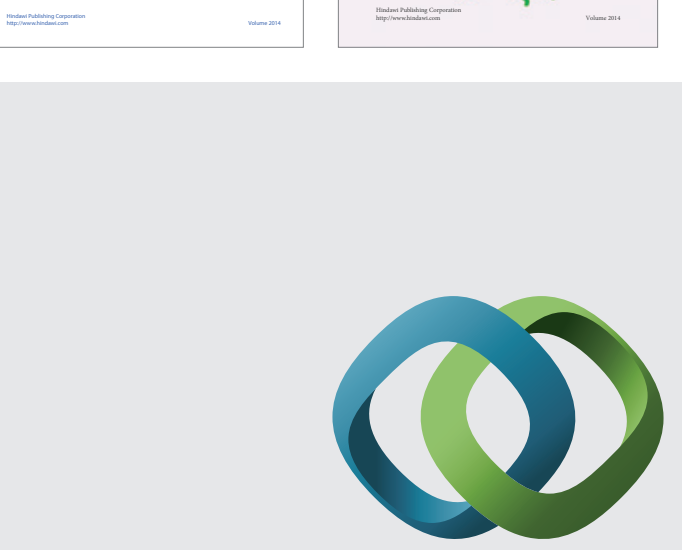

\section{Hindawi}

Submit your manuscripts at

http://www.hindawi.com
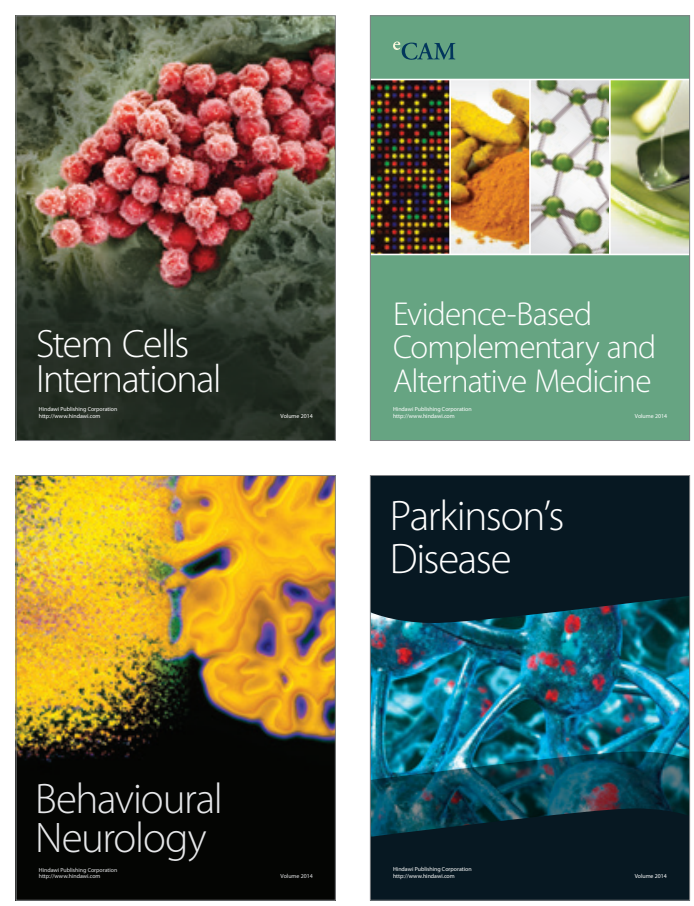

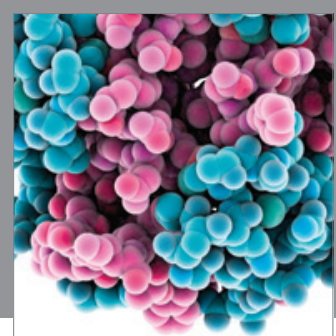

Journal of
Diabetes Research

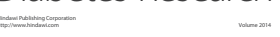

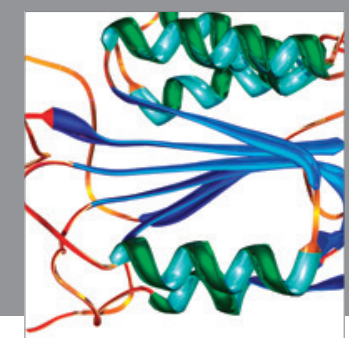

Disease Markers
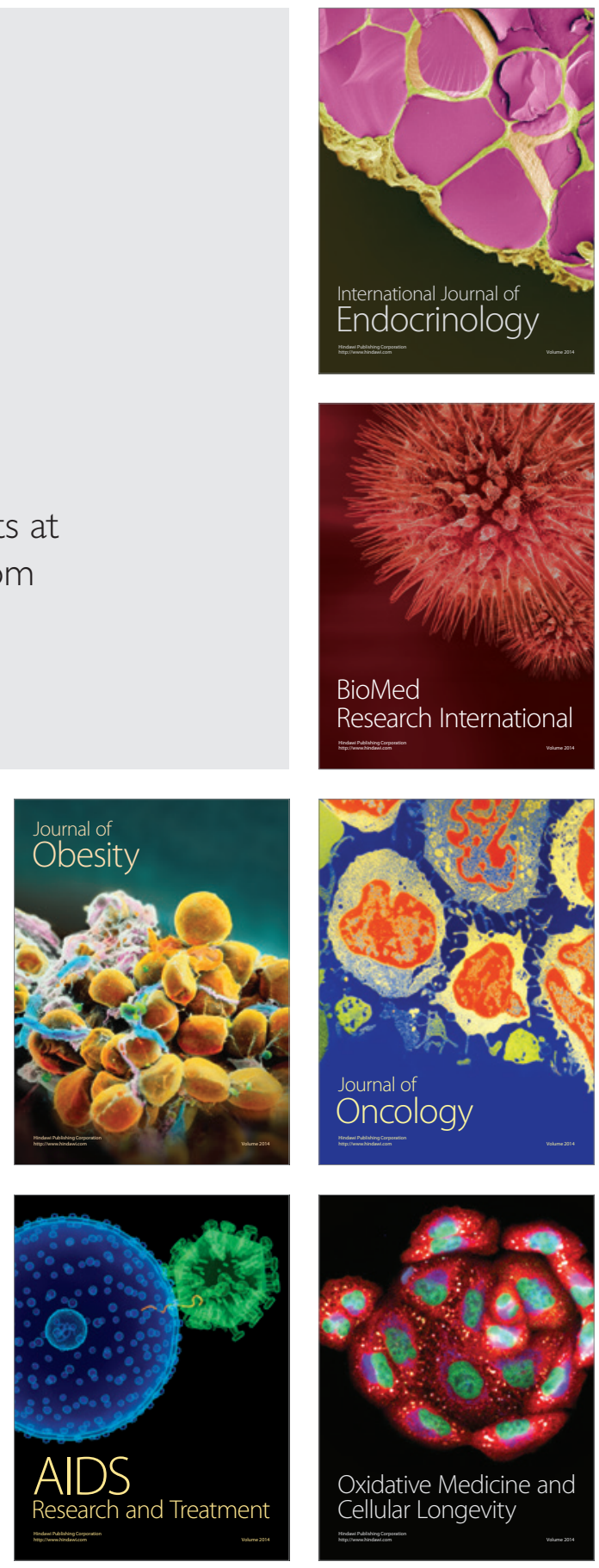